\title{
Exploiting pattern transformation to tune phononic band gaps in a two-dimensional granular crystal
}

\section{Citation}

Göncü, Fatih, Stefan Luding, and Katia Bertoldi. 2012. Exploiting pattern transformation to tune phononic band gaps in a two-dimensional granular crystal. The Journal of the Acoustical Society of America 131(6): EL475.

\section{Published Version}

doi:10.1121/1.4718384

\section{Permanent link}

http://nrs.harvard.edu/urn-3:HUL.InstRepos:11130513

\section{Terms of Use}

This article was downloaded from Harvard University's DASH repository, and is made available under the terms and conditions applicable to Open Access Policy Articles, as set forth at http:// nrs.harvard.edu/urn-3:HUL.InstRepos:dash.current.terms-of-use\#OAP

\section{Share Your Story}

The Harvard community has made this article openly available.

Please share how this access benefits you. Submit a story.

\section{Accessibility}


Exploiting pattern transformation to tune phononic band gaps in a two-dimensional granular crystal

\author{
F. Göncü and S. Luding \\ Multiscale Mechanics, \\ University of Twente, \\ PO Box 217, 7500 AE Enschede, Netherlands \\ f.goncu@utwente.nl \\ s.luding@utwente.nl
}

K. Bertoldi

School of Engineering and Applied Sciences,

Harvard University,

Cambridge, Massachusetts 02138, USA

bertoldi@seas.harvard.edu

Tuning band gaps in granular crystals 


\begin{abstract}
The band structure of a two-dimensional granular crystal composed of silicone rubber and polytetrafluoroethylene (PTFE) cylinders is investigated numerically. This system was previously shown to undergo a pattern transformation with uniaxial compression [Göncü et al. Soft Matter 7, 2321 (2011)]. The dispersion relations of the crystal are computed at different levels of deformation to demonstrate the tunability of the band structure which is strongly affected by the pattern transformation which induces new band gaps. Replacement of PTFE particles with rubber ones reveals that the change of the band structure is essentially governed by pattern transformation rather than particles' mechanical properties. (C)2012 Acoustical Society of America
\end{abstract}

PACS Numbers: 43.20.Mv, 43.40.At, 43.35.Gk 


\section{Introduction}

Wave propagation in materials with periodic microstructures? has been studied exten-

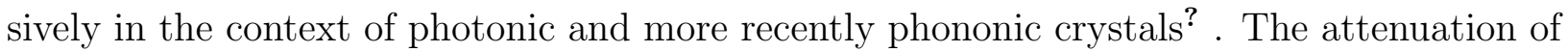
electromagnetic, acoustic or elastic waves in certain frequency ranges known as band gaps is an important feature of these materials which allows to use them as wave guides or filters? ?

Recent research has focused on the ability to control and tune the band gaps in phononic crystals. Several authors have reported? ? ?he modification and tuning of the band structure of phononic crystals with external fields. On the other hand, 1D granular crystals (i.e. periodic chains of particles) attracted increasing attention due to their non-linear dynamics arising from tensionless contacts and non-linear interactions between particles. Their nonlinear response can be tuned by changing the initial compression of the chain? ? ? , leading to the design of tunable acoustic lenses? and phononic band gap materials? . Moreover, theoretical studies? point out the possibility to control the band gaps of a periodic 2D granular crystal by introducing new periodicities in addition to existing ones.

Here, we investigate numerically the propagation of elastic waves in a $2 \mathrm{D}$ bi-disperse granular crystal composed of large (and soft) silicone rubber and small (and stiff) polytetrafluoroethylene (PTFE) cylinders? . In the undeformed crystal, particles are placed on two embedded square lattices (Fig. 1(a)). When the system is uniaxially compressed particles rearrange into a new periodic pattern? as illustrated in Fig. 1(b). We will show that the pattern transformation triggered by deformation can be effectively used to tune and transform the band gaps of the structure. The crystal under consideration consists of $5 \mathrm{~mm}$ radius silicone rubber and $2.5 \mathrm{~mm}$ radius PTFE particles. Material properties of silicone rubber are characterized by density $\rho_{r}=1.05 \times 10^{3} \mathrm{~kg} / \mathrm{m}^{3}$, Young's modulus $E_{r}=360 \mathrm{kPa}$, shear modulus $G_{r}=120 \mathrm{kPa}$ and longitudinal speed of sound $c_{r}^{l 0}=77.1 \mathrm{~m} / \mathrm{s}$, while for 
PTFE one has $\rho_{t}=2.15 \times 10^{3} \mathrm{~kg} / \mathrm{m}^{3}, E_{t}=1 \mathrm{GPa}, G_{t}=336.2 \mathrm{MPa}$, and $c_{t}^{l 0}=1350 \mathrm{~m} / \mathrm{s}$.

\section{Modeling}

Particles are modeled as 2D disks in a way similar to soft-particle Molecular Dynamics $(\mathrm{MD})^{?}$. They interact when they overlap and the forces in the normal contact direction are described by a non-linear contact force law (Fig. 1(d)):

$$
f(\delta)=k_{1} \delta+k_{2} \delta^{\alpha}
$$

where $\delta$ is the geometric overlap (see Fig. 1(c)). The parameters $k_{1}, k_{2}$ and $\alpha$ depend on the radii and mechanical properties of the particles in contact and their numerical values (listed in Table 1) are determined by fitting Eq. (1) to force-displacement data obtained from Finite Element Method (FEM) simulations of various contacts. For the sake of simplicity, tangential contact forces are modeled with a linear spring of stiffness $k_{t}$. Since a parametric study reveals that the magnitude of the tangential stiffness does not have a significant effect on the pattern transformation, here we assume $k_{t} / k_{n}=0.1481$ based on an estimate by

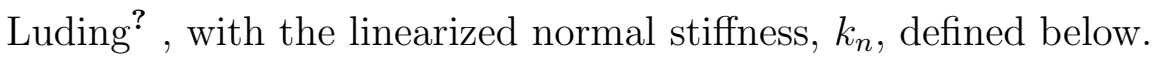

The propagation of elastic waves in infinite periodic lattices has been studied using techniques based on structural mechanics and FEM? ? . Following this approach two contacting particles $p$ and $q$ can be viewed as a finite element ${ }^{\text {? }}$ with the nodes located at the particle centers. Their interaction is then characterized by a stiffness matrix $\mathbf{K}^{p q}$ which relates the displacements and orientations (Fig. 1(c)) $\mathbf{U}^{p q}=\left[u_{x}^{p} u_{y}^{p} \theta^{p} u_{x}^{q} u_{y}^{q} \theta^{q}\right]^{\mathrm{T}}$ to the forces and torques acting on the particle centers $\mathbf{F}^{p q}=\left[f_{x}^{p} f_{y}^{p} \tau^{p} f_{x}^{q} f_{y}^{q} \tau^{q}\right]^{\mathrm{T}}$ such that $\mathbf{F}^{p q}=\mathbf{K}^{p q} \mathbf{U}^{p q}$ in the local coordinate system of the contact defined by the normal $\hat{\mathbf{n}}$ and tangent $\hat{\mathbf{s}}$, see Fig. 1(c). For a contact characterized by linear stiffnesses $k_{n}$ and $k_{t}$ in normal and tangential 
direction, respectively, $\mathbf{K}^{p q}$ is given by ${ }^{?}$ :

$$
\mathbf{K}^{p q}=\left[\begin{array}{cccccc}
k_{n} & 0 & 0 & -k_{n} & 0 & 0 \\
0 & k_{t} & k_{t} R^{p} & 0 & -k_{t} & k_{t} R^{q} \\
0 & k_{t} R^{p} & k_{t} R^{p} R^{p} & 0 & k_{t} R^{p} & k_{t} R^{p} R^{q} \\
-k_{n} & 0 & 0 & k_{n} & 0 & 0 \\
0 & -k_{t} & -k_{t} R^{p} & 0 & -k_{t} & -k_{t} R^{q} \\
0 & k_{t} R^{q} & k_{t} R^{p} R^{p} & 0 & -k_{t} R^{q} & k_{t} R^{q} R^{q}
\end{array}\right],
$$

where $R^{p}$ and $R^{q}$ are the radii of the particles. Note that, since we consider small amplitude perturbations of statically compressed particles with initial overlap $\delta_{0}$, Eq. (1) can be linearized as

$$
f(\delta) \approx f\left(\delta_{0}\right)+k_{n}\left(\delta-\delta_{0}\right)
$$

where $k_{n}=d f /\left.d \delta\right|_{\delta=\delta_{0}}$ is the linearized contact stiffness.

To compute the dispersion relation we consider an infinite crystal and solve the equations of motion for its periodic unit cell, disregarding effects due to finite systems with walls. Free harmonic oscillations are assumed and periodic boundary conditions are applied using Bloch's theorem? ? The final form of the equation of motion is of a generalized eigenvalue problem:

$$
\left[-\omega^{2} \mathbf{M}+\mathbf{K}\right] \mathbf{U}=0
$$

where $\omega$ is the radial frequency of the oscillations. $\mathbf{M}$ and $\mathbf{U}$ are the mass matrix and displacement vector of the unit cell, respectively and the global stiffness matrix $\mathbf{K}$ is assembled from the contributions of individual contacts according to the classical finite element assembly procedure. Note that although this approach assumes a fixed contact network and sliding between particles (i.e. friction) is neglected, it is still valid for this study since small amplitude perturbations superimposed to a given (finite) state of deformation are considered. 


\section{Results}

The dispersion diagrams for the 2D granular crystal at different levels of macroscopic nominal strain are provided in Fig. 2, clearly revealing the transformation of the band gaps with deformation. In the undeformed configuration the periodic unit cell of the crystal consists of a pair of rubber and PTFE particles arranged on a square lattice (Fig. 2(d)) and the structure possesses a phononic band gap for nondimensional frequencies $0.590<\tilde{\omega}<$ 0.823, where $\tilde{\omega}=\omega A /\left(2 \pi c_{r}^{l 0}\right)$ with $A=\left(\left\|\mathbf{t}_{1}\right\|+\left\|\mathbf{t}_{2}\right\|\right) / 2, \mathbf{t}_{1}$ and $\mathbf{t}_{2}$ being the lattice vectors.

At $15 \%$ compression the new pattern starts to emerge and the crystal has a unit cell composed of two pairs of rubber and PTFE particles (Fig. 2(e)). The structural transformation alters the dispersion relation of the crystal. Remarkably, a new band gap is open and the structure has now two band gaps at $0.141<\tilde{\omega}<0.419$ and $0.712<\tilde{\omega}<0.778$ (Fig. $2(\mathrm{~b}))$.

The transformation is complete when the PTFE particles touch (Fig. 2(f)). Figure 2(c) shows the corresponding band structure of the patterned crystal at $25 \%$ compression. The stiff contacts between PTFE particles leads to transmission and band gaps at much higher frequencies. At this level of deformation the structure is characterized by three band gaps in the intervals $0.142<\tilde{\omega}<0.545,0.885<\tilde{\omega}<3.557$ (partially shown in Fig. 2(c)) and $3.557<\tilde{\omega}<19.417$ (not shown in Fig. 2(c)).

Our previous study suggested that the qualitative nature of the pattern transformation

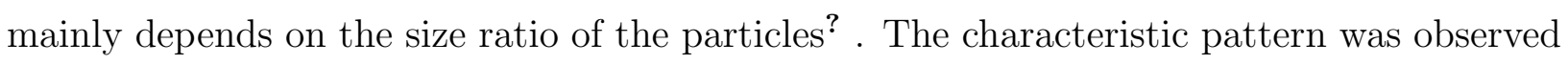
to form only when the size ratio $\chi=R_{\text {small }} / R_{\text {large }}$ of the small and large particles is in the range $\sqrt{2}-1 \leq \chi \leq 0.637$ and the transformation was found to be practically reversible around $\chi \approx 0.5$. Both FEM and MD simulations showed that the material properties of the particles do not play an essential role in the pattern transformation? . To investigate the 
effect of the material properties on the band gaps, we consider a crystal made entirely of rubber, replacing the $2.5 \mathrm{~mm}$ radius PTFE particles with rubber ones of the same size. The dispersion curves of the structure in the undeformed configuration and at $25 \%$ compression after pattern transformation are shown in Figs. 3(a) and 3(b), respectively, showing that the band structure is not affected qualitatively by the replacement. However, (i) the band structure is lowered due to the softer particles (Fig. 3(a)), and is significantly lowered at large strains (Fig. 3(b)) due to the absence of stiff contacts, (ii) the band gap of the undeformed rubber-rubber crystal (Fig. 3(a)) is wider than before (Fig. 2(a)), and (iii) in the deformed state of the soft structure, an additional narrow band gap is present at low frequencies.

Finally, we investigate the effect of the tangential stiffness of the contacts on the band structure by varying the ratio $k_{t} / k_{n}$ in the crystal composed of rubber-rubber particles, since the tangential stiffness depends on the material properties of the particles and can change when the crystal is further processed (e.g. by sintering? ). Increasing tangential stiffness $k_{t}$ leads to higher frequencies, but does not influence the pattern transformation. Focusing on the phononic properties, Figs. 3(c) and 3(d) show that both width and frequency of the band gaps increase with increasing tangential stiffness.

\section{Discussion and conclusion}

In this study of dispersion relations of regularly patterned granular crystals, we have considered the infinite, periodic case and neglected damping. The effect of finite size and viscous dissipation on phononic band gaps was studied by Jensen? , who reported that band gaps are still detectable. However, strong response peaks arise within the frequency range of theoretical band gaps due to localized motion near the boundary, and viscous damping smoothens the frequency response but the band gaps are still visible - unless damping is 
too strong. Therefore, we expect that the conclusions will not change for the finite size, dissipative versions of the granular crystals studied here.

In conclusion, we have shown that the band structure of a $2 \mathrm{D}$ bi-disperse soft granular crystal composed of large and small particles placed on two embedded square lattices can be modified considerably by deformation. The structural transformation triggered by compression leads to the opening of new band gaps. When translated to real frequencies the band gap marked with I in Fig. 3(b) falls between $5015.8 \mathrm{~Hz}$ and $5706.5 \mathrm{~Hz}$, which indicates that the crystal could be used as a tunable filter in the audible range, which makes such crystals promising candidates for applications in acoustics, when tunable band gap materials are needed.

\section{Acknowledgments}

F. Göncü and S. Luding acknowledge financial support from the Delft Center for Computational Science and Engineering. K. Bertoldi acknowledges the support from Harvard Materials Research Science and Engineering Center and from the Kavli Institute at Harvard University. 
Göncü, JASA-EL 
Göncü, JASA-EL

Table 1. Numerical values of contact force parameters $k_{1}, k_{2}$ and $\alpha$ for pairs of silicone rubber (SR) and PTFE particles.

\begin{tabular}{lccr}
\hline \hline & $k_{1}[\mathrm{~N} / \mathrm{mm}]$ & $k_{2}\left[\mathrm{~N} / \mathrm{mm}^{\alpha}\right]$ & $\alpha$ \\
\hline $\mathrm{SR}^{a}-\mathrm{SR}^{a}$ & 1.3459 & 0.1264 & 2.9793 \\
$\mathrm{SR}^{a}-\mathrm{PTFE}^{b}$ & 2.5197 & 0.2217 & 3.3028 \\
$\mathrm{PTFE}^{b}-\mathrm{PTFE}^{b}$ & 3468 & 1706.9 & 2.8147 \\
$\mathrm{SR}^{a}-\mathrm{SR}^{b}$ & 1.3992 & 0.4921 & 3.1357 \\
$\mathrm{SR}^{b}-\mathrm{SR}^{b}$ & 1.1018 & 0.4372 & 2.3877 \\
\hline \hline
\end{tabular}

${ }^{a} R=5 \mathrm{~mm}$

${ }^{b} R=2.5 \mathrm{~mm}$ 


\section{List of Figures}

Fig. 1 (Color online) (a) Initial undeformed granular crystal and (b) patterned configuration after $25 \%$ uniaxial compression, adapted from Göncü et al. ${ }^{\text {? }}$. (c) Sketch of two particles in contact showing displacements and the geometric overlap $\delta$. (d) Normal contact force as a function of the overlap for a pair of (5 mm) silicone rubber and $(2.5 \mathrm{~mm})$ PTFE particles. . . . . . . . . . . . 13

Fig. 2 (Color online) Top: Dispersion curves of the bi-disperse granular crystal composed of large rubber $(5 \mathrm{~mm})$ and small PTFE $(2.5 \mathrm{~mm})$ particles with tangential stiffness $k_{t}=0.1481 \times k_{n}$ at (a) $0 \%$, (b) $15 \%$ and (c) $25 \%$ uniaxial compression. The vertical axes represent the non-dimensional frequencies $\tilde{\omega}=\omega A /\left(2 \pi c_{r}^{l 0}\right)$ with $A=\left(\left\|\mathbf{t}_{1}\right\|+\left\|\mathbf{t}_{2}\right\|\right) / 2$. Bottom: Unit cells, lattice vectors $\mathbf{t}_{1}$ and $\mathbf{t}_{2}$ and the first Brillouin zones of the crystal at (d) $0 \%$, (e) $15 \%$ and (f) $25 \%$ uniaxial compression. The shaded areas indicate the irreducible parts of the Brillouin zones. . . . . . . . . . . . . .

Fig. 3 Dispersion relation of a soft granular crystal made of rubber particles in the (a) undeformed and (b) patterned state (at $25 \%$ compression) with $k_{t} / k_{n}=$ 0.1481. Evolution of the band gaps in the (c) undeformed and (d) patterned (band gaps marked by I, II and III in Fig. ??(b)) soft granular crystal as function of the stiffness ratio $k_{t} / k_{n}$. 
Göncü, JASA-EL 
Göncü, JASA-EL
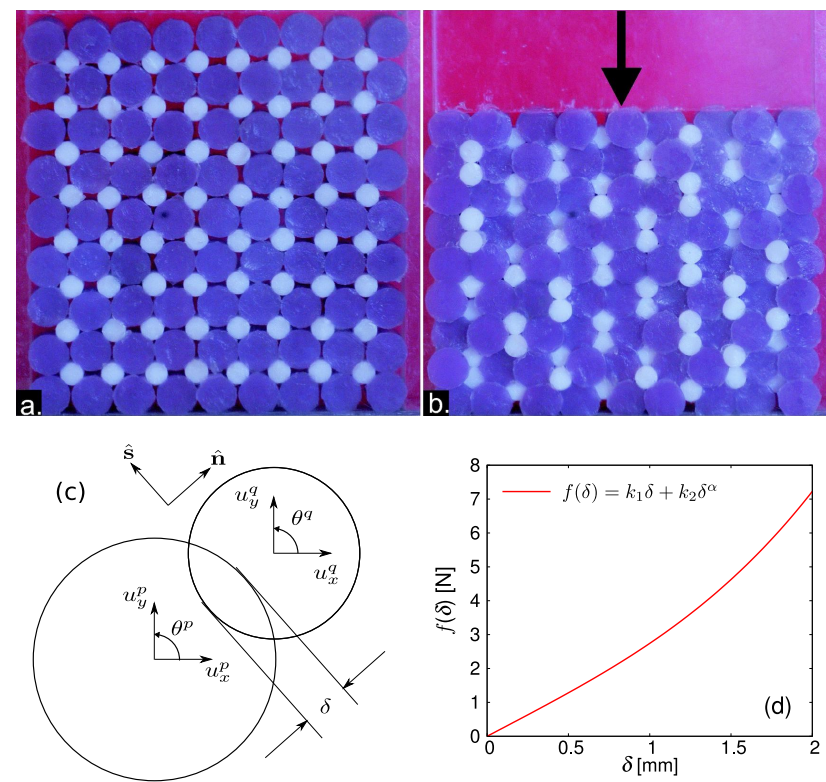

Fig. 1. 
Göncü, JASA-EL
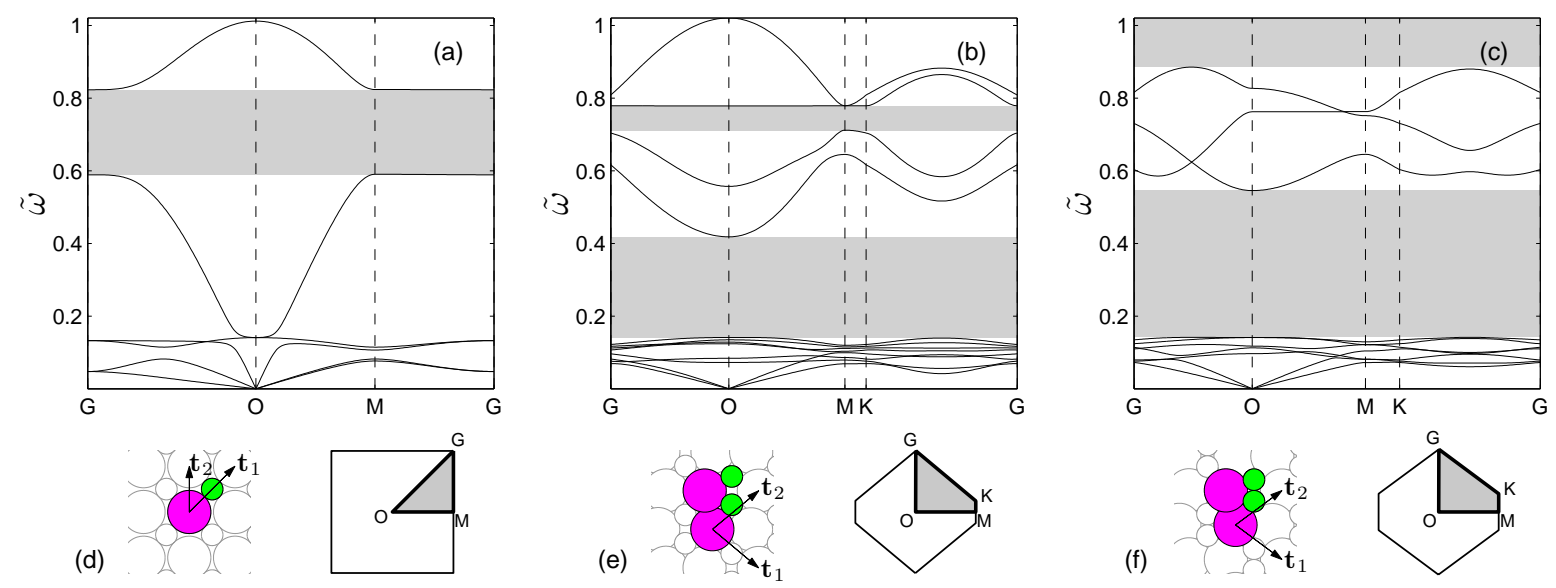

Fig. 2. 
Göncü, JASA-EL
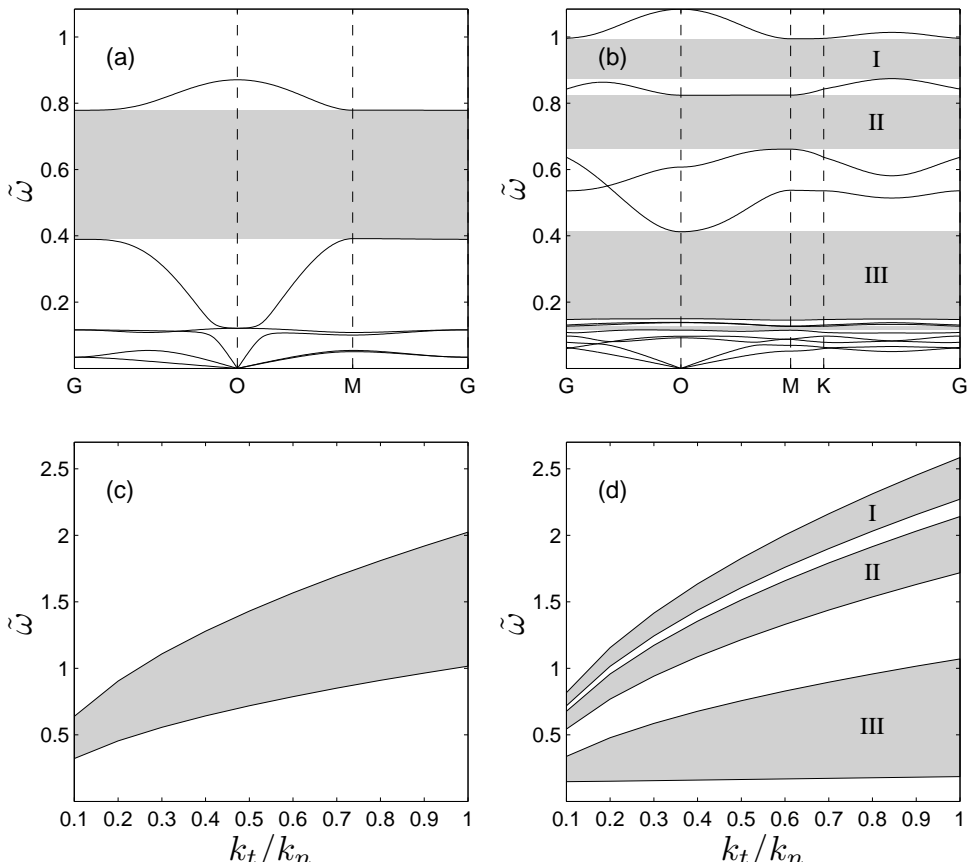

Fig. 3. 